\title{
Bienestar colectivo comunal a nivel de microcuencas, en comunidades campesinas, provincia de Andahuaylas, Región Apurímac
}

\author{
Communal collective well-being at micro-watershed level in peasant \\ communities, province of Andahuaylas, Apurimac Region
}

\section{${ }^{1}$ Felipe Valle Díaz}

\section{RESUMEN}

La investigación tuvo como objetivo principal describir el estado de bienestar colectivo comunal logrado para los años 2013 y 2017 a nivel de las microcuencas en la provincia de Andahuaylas, teniendo en cuenta como dimensiones: recursos naturales, producción orgánica y gobernanza comunal. El tipo de investigación fue no experimental, con un diseño descriptivo-comparativo. Las unidades de análisis territoriales comparadas poseen características similares respecto a clima, suelo, relieve y organización comunal. La toma de datos se realizó a una muestra intencionada formada por las autoridades. Entre las conclusiones se encuentra que, los indicadores (ratios) de beneficio monetario de la producción orgánica precisan que los costos fueron cubiertos, existiendo una utilidad a pesar de la variabilidad de precios y el costo de la mano de obra diversa. Los indicadores descendieron para el año 2017 respecto al año 2013. La comercialización se centró en vínculos y contratos de comercio justo. Con respecto a la cultura de uso de recursos naturales, esta mostró indicadores porcentuales heterogéneos; por ejemplo, las coberturas de los servicios de saneamiento básico se expandieron, ofreciéndose agua entubada y clorada, además de desagüe. El año 2013 se tuvo una cobertura aceptable; mientras que, en el año 2017, la cobertura fue próxima a lo óptimo. Además, las áreas naturales comunales están protegidas (manteniéndose año a año), existen planes de conducción por plantación forestal, pastos naturales, frutales, entre otras acciones. La gobernanza comunal mostró diferencias, específicamente se puede afirmar que los acuerdos, consensos y tratos se respetan; además, son legitimados por los miembros de la comunidad campesina. El año 2013, en la comunidad campesina Huayana, se evidenció un debilitamiento en su organización, revirtiéndose en el año 2017. En el resto de comunidades campesinas se redujo el debilitamiento de la organización comunal. Los resultados afirman que existe un estado de mejora progresiva en el bienestar colectivo comunal por cada microcuenca y comunidad campesina, presentando diferencias considerables. Se concluye que el nivel de bienestar colectivo es fuerte a pesar del contexto dado.

Palabras claves: Bienestar, comunidad campesina, gobernanza, producción, recursos naturales.

\section{ABSTRACT}

The research described the communal collective welfare state achieved for the years 2013 and 2017. The type of research is nonexperimental, descriptive-comparative design. The territorial analysis units compared have similar characteristics regarding climate, soil, relief, communal organization. The data collection was made to an intentional sample formed by the authorities.

The indicators (ratios) of monetary benefit of the organic production, specify that the costs are covered, there is a utility in spite of the price variability and the cost of the diverse workforce. The indicators decreased for the year 2017 with respect to the year 2013 ; the marketing focuses on links and contracts in fair trade.

The culture of use of natural resources, show heterogeneous percentage indicators, the coverage of basic sanitation services was expanded for the case chlorinated piped water and drainage, for the year 2013 an acceptable coverage, for the year 2017 a coverage close to the optimum. The communal natural areas are protected, the proportion of care of the protected natural area is maintained, year after year, between the $50 \%$ to $100 \%$ of the territory, there are also driving plans by forest plantation, natural pastures, fruit trees; In addition to maintaining the practice of organicity with an age between 30 to 50 years.

The communal governance, shows differences, specifically we can affirm that the agreements, consensus, treatment; they are respected, legitimized by the members of the peasant community. For the year 2013, the Huayana peasant community showed a weakening in its organization, reversing for 2017, in the rest of the Comunidades Campesinas the weakening of the communal organization was reduced.

The results affirm that there is a state of progressive improvement in the communal collective welfare for each microbasin and rural community, presenting considerable differences. It is concluded that the level of collective welfare is strong, greater integration, under the given context.

Keys words: Well-being, natural resources, production, governance, peasant community.

${ }^{1}$ Departamento Académico Ciencias Empresariales. Universidad Nacional José María Arguedas. Andahuaylas-Perú.

E-mail: fvalle@unajma.edu.pe

Presentado: 24/01/19, Aprobado: 15/04/19 


\section{INTRODUCCIÓN}

La Región Apurímac, ubicada en los contrafuertes andinos de la sierra sur central del Perú, presenta una geografía con forma de papel arrugado, un relieve accidentado con cortes o inclinaciones de $20^{\circ}$ a $39^{\circ}$ grados y con ciertas áreas erosionadas de suelo; la altimetría fluctúa entre los 2400 a $5500 \mathrm{msnm}$. La población fue calificada en situación de extrema pobreza monetaria, ubicada entre el tercer grupo robusto en el año 2013, cuyos límites fueron $5.7 \%$ y $7.5 \%$; y en el año 2017 fue ubicada en el mismo grupo, cuyos límites fueron $4.7 \%$ y $6.5 \%$ (Instituto Nacional de Estadística e Informática Perú, 2018). Cuenta con suficiente capacidad de reserva de agua, frontera de reserva forestal, prácticas ancestrales perennes, saberes culturales enraizados en cultivos como maíz, papa, trigo y cebada, que sostienen la práctica agropecuaria, actualmente.

La investigación se centra en conocer el bienestar colectivo comunal los años 2013 y 2017, a partir de la interrogante preliminar: ¿cuáles han sido las variables que incidieron y están relacionadas respecto al nivel de bienestar colectivo alcanzado, y si entre ambos periodos sucedieron algunos cambios considerables?

Para conocer el bienestar colectivo comunal es necesario tener en cuenta tres dimensiones.

La primera dimensión es la cultura de uso de los recursos naturales; manifestada por las formas, costumbre de cuidado, control y conservación del recurso suelo (área agrícola, pecuaria y forestal), sin tener un impacto alto en el medio ambiente (Ostrom, 2009). Ello no vulnera sus medios de existencia circundantes, además contiene el aspecto del uso adecuado del recurso hídrico para el saneamiento básico, necesario para una adecuada salud, cuya correspondencia es la preservación y cuidado del agua (MINAM, 2011).

Otra dimensión es la producción orgánica, referida al aprovechamiento del suelo y explotación racional, manifestado físicamente en volúmenes de producto o cosecha, transformado monetariamente en soles peruanos.
La dimensión gobernanza se refiere a la forma de consensuar, acatar y comprometerse con los acuerdos comunales aprobados en asamblea comunal; los cuales sostienen el quehacer diario y fortalecido en costumbres, como la práctica de la reciprocidad.

La variable bienestar colectivo está conformada por estas dimensiones, que están ligadas y tienen sus propio procesos y estructura, cuyos comportamientos nos permite describir el estado de bienestar colectivo actual.

Dentro del contexto de estudio, las comunidades campesinas se encuentran contenidas y sostenidas en el marco legal vigente, Ley General de Comunidades Campesinas (Ley $\mathrm{N}^{\circ} 24656,1987$ ) y Ley del Deslinde y Titulación de Territorios Comunales (Ley $\mathrm{N}^{\circ} 24657,1987$ ); las cuales formalizan, protegen y resguardan la organización y territorio comunal. Asimismo, las comunidades campesinas están contenidas explícitamente en la Constitución Política del Perú (1993). Su contexto organizacional se centra en la administración territorial por familias, quienes en asamblea deciden la organización, explotación y conservación del territorio comunal. Poseen medios de subsistencia limitados y aprovechados en forma racional al máximo, los cuales se regulan con la aprobación de la mayoría en asamblea comunal; si ello sucede, no se conoce hasta qué punto el bienestar es fuerte, moderado o bajo y además si es aprovechado por todos. Esta regulación se sostiene en acuerdos aprobados en asamblea general, lo cual permite una interdependencia entre los agentes económicos $\mathrm{y}$, del mismo modo, hacia las condiciones ambientales que los rodean.

Uno de los tantos acuerdos, desarrollados (como otros no desarrollados), es la práctica de producción orgánica, que se logra en la realización de productos; manifestada por el beneficio de la decisión tomada. Asimismo, el criterio y aprobación de preservar recursos naturales diversos al interior de los territorios comunales es característico, como el de mantener medios de existencia para generaciones futuras. Para los años de estudio, 2013 y 2017, la legislación se mantuvo constante, es decir no existió modificación alguna en la Ley 24656 y la Ley 24657. 
Los periodos establecidos para elección de autoridades comunales son cada dos años. Las funciones solo alcanzan a la administración, aprovechamiento del territorio comunal, preservación de recursos naturales y buenas prácticas de producción orgánica. No tienen injerencia de funciones en la administración pública de los centros poblados menores.

La información sobre el manejo de los recursos naturales al interior de los territorios comunales, en revistas especializadas y páginas web de instituciones ligadas al sector agrario para el año 2013, es exigua. Además, que esta información es difícil de obtener, porque una considerable cantidad de unidades de análisis están dispersas dentro del territorio comunal; y que, en muchas ocasiones por su extensión superficial comprende más de un distrito.

El sistema de información ambiental regional (siar.regionapurimac.gob.pe) monitorea clima, temperatura, suelo y residuos sólidos. Además, integra información de cuentas nacionales, estadísticas económicas, sociales, normatividad y mapas interactivos. Dicha información es recabada a partir de otras instituciones asociadas, ligadas mediante un convenio institucional de cooperación. Revisada la página web, siar.regionapurimac, se constató que no precisa información específica a nivel de microcuenca y las estadísticas a nivel comunal son inexistentes; no sirven de referencia, menos para una comprobación posterior. Asimismo, es limitada para justificar estadísticamente la propuesta de políticas u otros modelos de intervención específicos de acuerdo a la realidad.

La oficina de Información Agraria Andahuaylas empezó su proceso de fortalecimiento desde el año 2015 , consolidándose el año 2017. Fue desde esta entidad donde se pudo extraer datos a nivel de agencias agrarias, ubicadas por grupo de distritos representativos ( 2 a 3 distritos juntos), no existiendo a nivel de territorio comunal. Asimismo, el Sistema de Focalización de Hogares (SISFOH) contiene una base de datos a nivel distrital respectos a familias ubicadas en zonas de extrema pobreza, pero cuyas estadísticas no están procesadas a nivel de territorio comunal (algunos distritos contienen desde 2 a 4 territorios comunales, un territorio comunal abarca mínimamente una superficie de 900 has a más) igual sucede con la Encuesta Nacional de Hogares (ENAHO). Al no poseer datos suficientes, la información final es no confiable y por el contexto, que contiene una diversidad cultural, social y heterogénea, se tuvo que iniciar realizando diagnósticos. Lo más resaltante es que la sistematización de datos para el año 2017 arrojó diferencias considerables en varias dimensiones con respecto al año 2013, por lo cual; en lo similar existe evidencia mínima en dimensiones.

La pregunta general de la presente investigación es ¿cuál es el estado de bienestar colectivo comunal logrado a nivel de las microcuencas: Pomacocha-UmamarcaHuayana, Chiara-Santiago de Yaurecc-San Miguel de Chaccrampa, Matapuquio-Belen Anta-Santiago de Paucar, provincia Andahuaylas, Perú, 2013 y 2017 ?

Las preguntas específicas fueron: ¿cuál es el estado de la cultura de uso de recursos naturales logrado a nivel de microcuencas? ¿cuál es el estado de la producción orgánica logrado a nivel de micro cuencas? ¿cuál es el estado de la gobernanza comunal logrado a nivel de microcuencas?

El objetivo general de la investigación fue describir el estado de bienestar colectivo comunal logrado a nivel de las microcuencas: Pomacocha-Umamarca-Huayana, ChiaraSantiago de Yaurecc-San Miguel de Chaccrampa y Matapuquio-Belén AntaSantiago de Paucar, provincia Andahuaylas, Perú, 2013 y 2017.

Los objetivos específicos se ciñen a describir el estado de la implantación de una cultura de uso de recursos naturales logrado a nivel de microcuencas; describir el estado de la producción orgánica logrado a nivel de microcuencas, y describir el estado de la gobernanza comunal logrado a nivel de microcuencas.

\section{MARCO CONCEPTUAL}

Entendemos por bienestar colectivo comunal a la relación entre la cultura de organicidad implantada, la producción orgánica y la gobernanza comunal. Además, como la 
relación y articulación que existe entre el manejo o distribución, el uso o explotación adecuado de recursos naturales, el beneficio e impacto de la producción orgánica, la aprobación, cumplimiento y respeto a los acuerdos comunales respecto al uso sostenible de recursos naturales.

Con respecto al uso adecuado de recursos naturales, según Garza (2004), la cultura en el cuidado del ambiente y recursos naturales es aquella que se sostiene en las creencias, valores, comportamientos, que se inculcan en la sociedad, generando un comportamiento proactivo de protección, preservación relacionando al contexto y cultura donde se ubica el hombre.

Entonces, entendemos por cultura de uso adecuado de los recursos naturales a la programación de las costumbres, para la explotación y preservación de los recursos naturales al interior del territorio comunal Ostrom (2009). Siendo estos: Uso y preservación del agua, evacuación de desechos líquidos, áreas naturales protegidas y cumplimiento de acuerdos sobre el uso de recursos naturales.

Por otro lado, la producción orgánica comprende el ratio de los ingresos de la producción orgánica según cultivo y tipo de contrato, ingresos de la producción tradicional, entre los costos de la producción orgánica según cultivo y tipo de contrato, costos de la producción tradicional (Figueroa, 2006).

Además, según la FAO (2001), la producción orgánica se sostiene en la agricultura orgánica, porque ella parte de realizar prácticas amigables con la naturaleza, abandonando plaguicidas, por el uso y transformación de desechos orgánicos. Similar al concepto de la USDA, departamento de agricultura de Estados Unidos (1980).

Con respecto a la gobernanza comunal, la Organización de Naciones Unidas - ONU (2016), indica que: "La gobernanza democrática fomenta el desarrollo, dedicando su energía a influir en tareas como la erradicación de la pobreza, la protección del medio ambiente, garantizar la igualdad entre los géneros y proporcionar los medios de subsistencia sostenibles" (p.1).
Asimismo, según el Banco Interamericano de Desarrollo - BID (2009), se define Gobernanza a la diferencia con el gobierno. El gobierno incluye la capacidad institucional en la gestión y administración pública con participación de los diferentes actores. La gobernanza alude a la habilidad para coordinar y promover políticas, programas y proyectos que representen los intereses de todos los actores locales, públicos y privados.

Para finalizar, la gobernanza comunal está acotada a la cantidad y grado de aprobación, cumplimiento, legitimidad de los acuerdos comunales; coordinados respecto al uso sostenible de recursos naturales y la decisión de realizar producción orgánica.

\section{METODOLOGÍA}

El tipo de investigación fue descriptiva, sostenida en que no se realiza manipulación de las variables, se toman los datos tal como se encuentran de las variables en estudio, no se pretende determinar influencia ni efecto entre las variables (Palomino, 2011).

El diseño de la investigación fue descriptivocomparativo. La investigación se forma desde una estructura descriptiva hacia una comparativa respecto a las microcuencas. $\mathrm{La}$ primera en base a cuadros estadísticos y la segunda en base de matrices comparativas, donde se establecen semejanzas y diferencias (Palomino, 2011).

\section{VARIABLES Y DIMENSIONES}

La variable es: Bienestar colectivo comunal. Dentro de las dimensiones se tiene la producción orgánica, cultura de uso del recurso natural y gobernanza comunal.

\section{MÉTODO}

El método usado es el descriptivo, partimos de hechos y procesos que son observables, procedimientos referenciados, que contribuyen a revelar características, diferencias, semejanzas, relaciones de los hechos; obtenidos a partir de las unidades de análisis y conocimientos utilizados (Palomino, 2011). 
Dentro de las técnicas utilizadas estuvo la encuesta, que se apoyó en una ficha con preguntas cerradas y estructuradas. Se aplicó una ficha para cada dimensión de estudio que compone el bienestar colectivo comunal. La entrevista, que se apoyó en una ficha, sirvió para profundizar el problema de estudio y cruzar datos respecto a la dimensión gobernanza y cultura de uso del recurso agua.

\section{ANÁLISIS DEL TERRITORIOY MUESTRA}

La población estuvo compuesta por tres microcuencas con características similares: de relieve accidentado, topografía entre 15 a 35 grados, climas semejantes, que contienen tres comunidades campesinas con cultivos manejados en forma tradicional, con calificación de extrema pobreza y organización comunal activa.

En la Figura 1 se puede observar la ubicación de las microcuencas en la provincia de Andahuaylas.

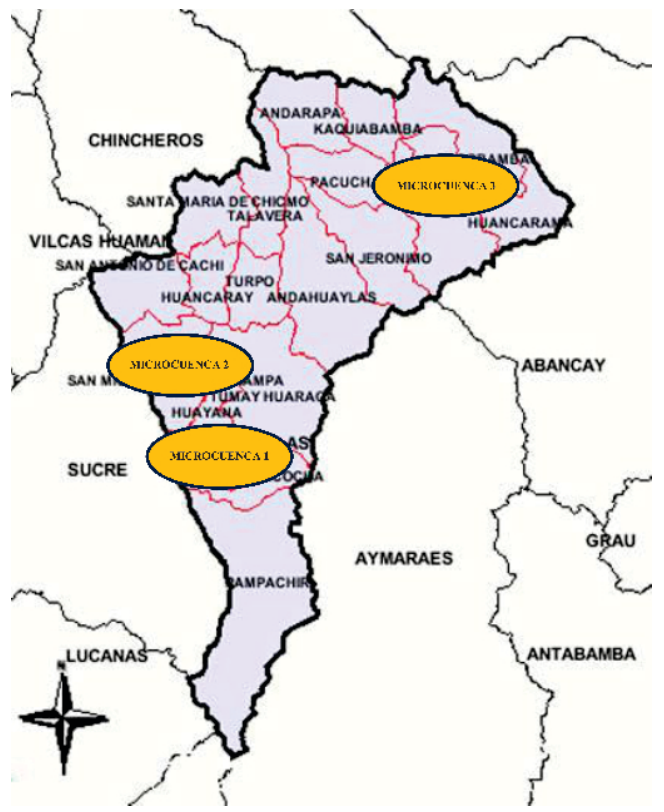

Figura 1. Microcuencas de intervención en el trabajo de investigación.

Fuente: INEI - 2016
La muestra fue intencionada y no probabilística; se obtuvo la información por parte de las autoridades actuales y anteriores.

En las Figuras 2 y 3 pueden observarse las micro cuencas desde una vista satelital.

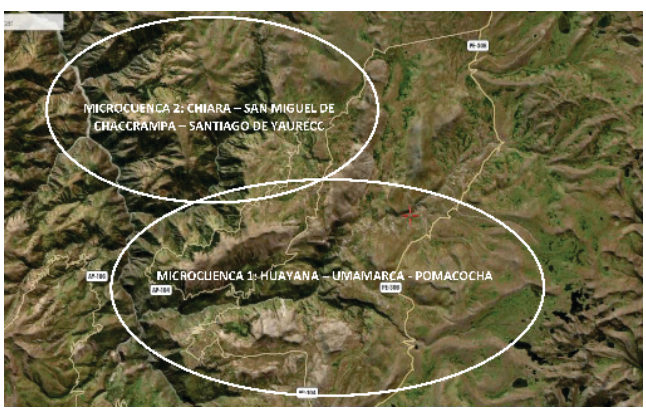

Figura 2. Foto satelital microcuenca 1: HuayanaUmarmaca-Pomacocha. Microcuenca 2: Chiara - San Miguel de Chaccrampa - Santigo de Yaurecc

Fuente: Apple TM+Google TM.

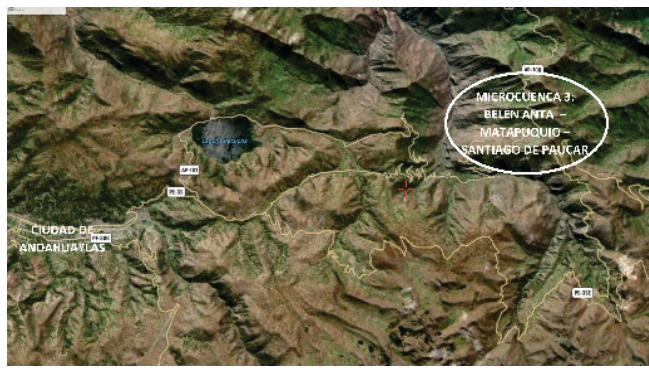

Figura 3. Foto satelital microcuenca 3: Belén Anta - Matapuquio - Santiago de Paucar.

Fuente: Apple TM + Google TM.

\section{RESULTADOS LA INVESTIGACIÓN}

Para el objetivo general, los resultados descritos y comparados demuestran que existe una mejora y un estado fuerte; que no es homogénea en los territorios comunales campesinos a nivel de microcuencas. Se evidenció diferencias considerables.

En la Tabla 1 se presentan los resultados descriptivos finales de la investigación. Por otro lado, en la Tabla 2 se presentan los comparativos de resultados finales. 


\section{Tabla 1}

\section{Resultados descriptivos finales}

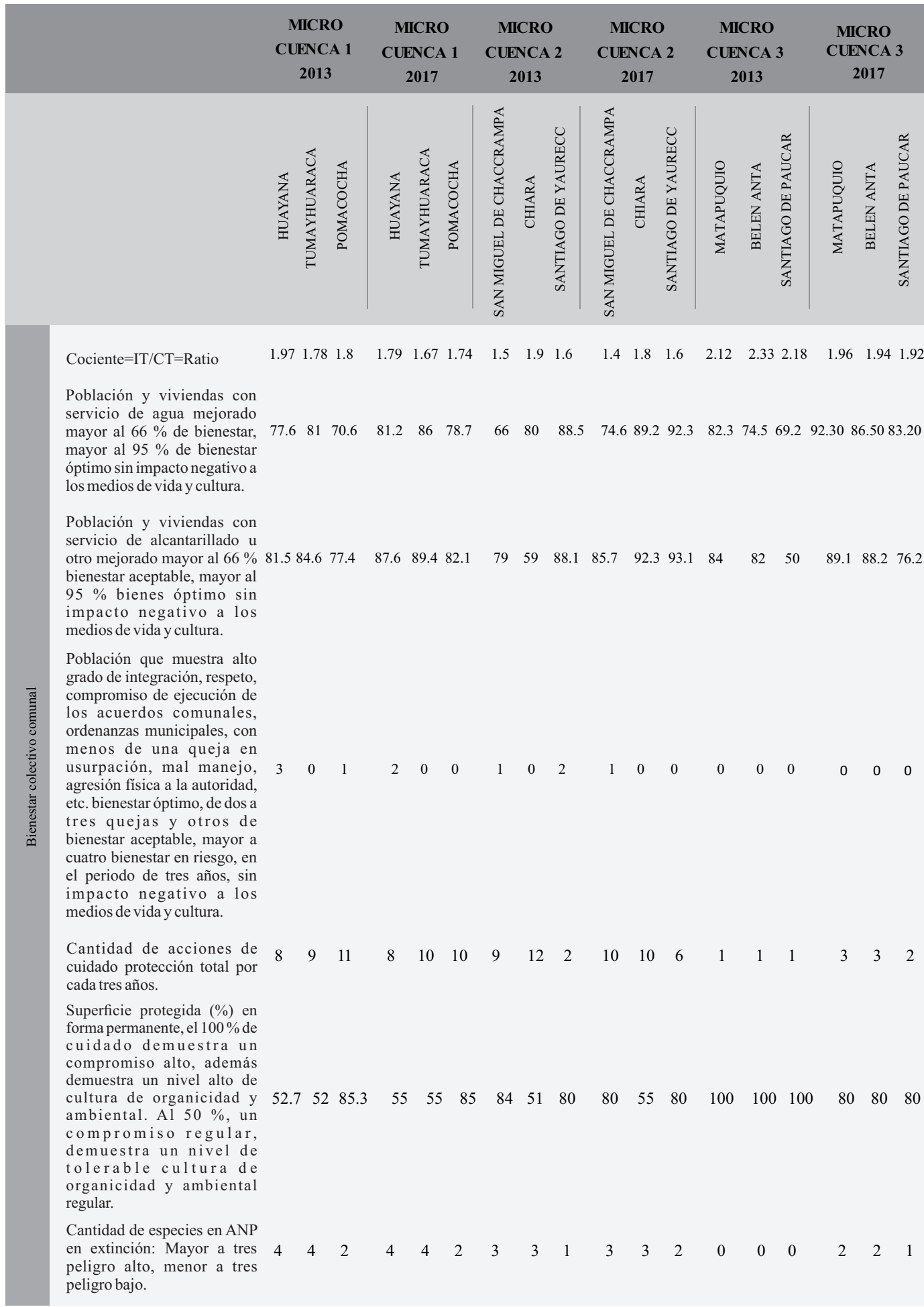


Tabla 2

Comparativo de resultados finales

\begin{tabular}{|c|c|c|c|c|c|c|c|c|c|}
\hline $\begin{array}{c}\text { yVDกVd } \\
\text { gם ODVILNVS }\end{array}$ & 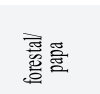 & 뮬 & 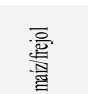 & $\approx$ & 앙 & 骂 & $\bar{s}$ & 韋 & 음 \\
\hline VLNV Nज़TGg & 莺隠 & 뮬 & $\cong$ & 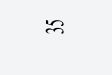 & \& & 咆 & $\bar{s}$ & 悪 & $\cong$ \\
\hline OInठ̊กdVLVW & 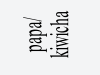 & 뮬 & 믈 & $\approx$ & f & 贾 & 임 & 豞 & $\cong$ \\
\hline $\begin{array}{c}\text { yVDกVd } \\
\text { घ ODVILNVS }\end{array}$ & 喼 & $\begin{array}{l}\text { 量 } \\
\text { 善 }\end{array}$ & $\begin{array}{l}\text { 을 } \\
\text { 量 } \\
\text { 竞 }\end{array}$ & e & 商 & 焉 & 욤 & 营 & 욤 \\
\hline VLNV NG̣̈TAg & 墨 & 믈 & 임 & \& & e & 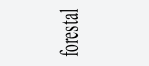 & 임 & 鸹 & 임 \\
\hline 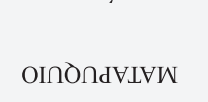 & 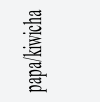 & 뮬 & 뮵 & 하 & 요 & 虽 & 욤 & 㤩 & 욤 \\
\hline 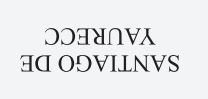 & 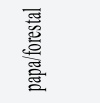 & 뮴 & 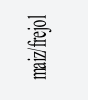 & $\approx$ & \& & 要 & $\bar{\infty}$ & 蹄 & 음 \\
\hline У४УНว & 踣 & 뮵 & 葾 & $\approx$ & $\therefore$ & 骂 & 임 & 壖 & 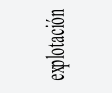 \\
\hline $\begin{array}{l}\text { VdWVУכวVHD } \\
\text { Gd TAกDIW NVS }\end{array}$ & 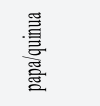 & 뮬 & 总 & $\approx$ & F & 骂 & $\bar{\infty}$ & 嘉 & 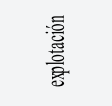 \\
\hline 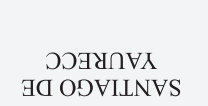 & 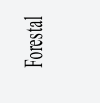 & 뮬 & 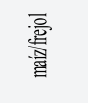 & e & $\approx$ & 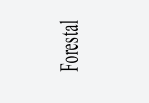 & $\dot{\infty}$ & 总 & 운 \\
\hline VyทHว & 善 & 륩 & 㖺 & e & 善 & 嘿 & $\cong$ & 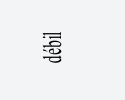 & ะ 总 \\
\hline 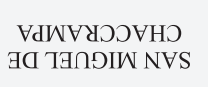 & 恚 & 률 & 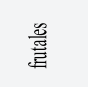 & 8 & e & 跑 & 욤 & 总 & $\begin{array}{l}\text { 葶 } \\
\text { 言 }\end{array}$ \\
\hline & 吾涪 & 急焉 & 푤 & $\approx$ & $\approx$ & 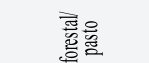 & $\bar{m}$ & 总 & $\cong$ \\
\hline $\begin{array}{c}\text { VHDODVWOd } \\
\text { VDVYV } \\
\text { กHXVWחL }\end{array}$ & 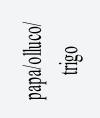 & $\begin{array}{l}\text { 意 } \\
\text { 量 }\end{array}$ & 咅 & $\therefore$ & 8 & 焉 & $\cong$ & 営 & 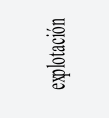 \\
\hline VNVגVחH & 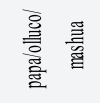 & 要总 & 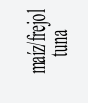 & $\approx$ & 8 & 要 & के & 嘉 & 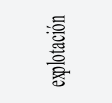 \\
\hline 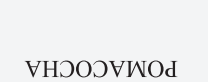 & 襃:兽 & 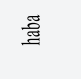 & 뮬 & e & $\approx$ & 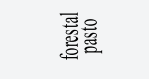 & $\overrightarrow{5}$ & 鸹 & 욤 \\
\hline $\begin{array}{c}\text { VDVYY } \\
\text { กHXVWกL }\end{array}$ & $\begin{array}{l}\text { 总 } \\
\text { 总 } \\
\text { 总 }\end{array}$ & $\begin{array}{l}\text { 를 } \\
\text { 善 }\end{array}$ & 뮵 & 屌 & 数 & 폴 & 임 & 놀 & ₹ \\
\hline VNVגVחH & 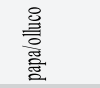 & 㗊 & 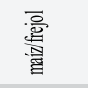 & 요 & $\approx$ & 蕊 & $\bar{s}$ & 骂 & 产 \\
\hline 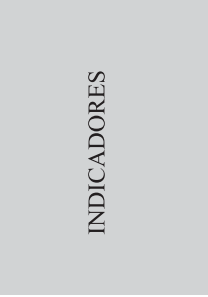 & 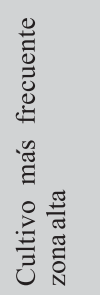 & 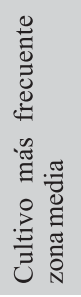 & 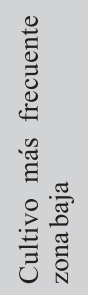 & 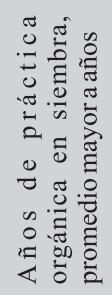 & 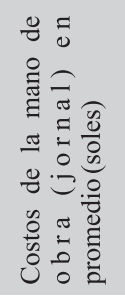 & 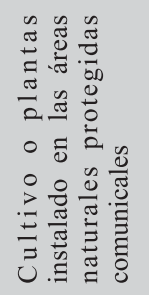 & 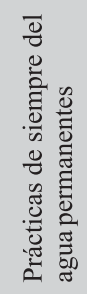 & 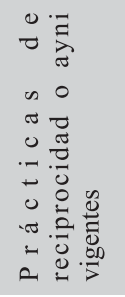 & 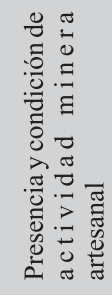 \\
\hline
\end{tabular}


Para el caso de la producción orgánica, esta se muestra en los ratios de beneficio monetario de la producción orgánica. Demuestran que los costos son cubiertos y existe una utilidad, a pesar de la variabilidad de precios y costo de la mano de obra diversa. Los ratios de beneficio monetario de la producción orgánica cayeron para el año 2017 respecto al año 2013, el rango de variación es 1.4 a 2.33.

Para el caso de la dimensión cultura de uso de recursos naturales, los servicios de saneamiento básico han mejorado en expansión de la cobertura en agua entubada y clorada, y desagüe para beneficio de la población rural. Es notorio que los indicadores porcentuales son heterogéneos, no extremos; para el año 2013 el estado de bienestar era aceptable, para el año 2017 el estado de bienestar fue próximo al óptimo. Las áreas naturales comunales son protegidas, sigue existiendo diferencias en cuanto al porcentaje que mantienen en protección por cada comunidad campesina. Se mantiene la proporción de cuidado del área natural protegida, tanto para el año 2013 y el 2017, rango entre el $50 \%$ al $100 \%$ del territorio. Existe un plan de conducción aprobado por cada plantación forestal, plantación de pastos naturales y frutales. Mantienen la práctica de organicidad que tienen una antigüedad superior a 30 años, esta es constante y se refleja en los datos de los años 2013 y 2017.

Para el caso de la dimensión gobernanza comunal, los resultados arrojan heterogeneidad, donde específicamente podemos afirmar que los acuerdos, consensos, tratos se respetan y legitiman por los miembros de la comunidad campesina. Para el año 2013, el caso más resaltante fue la comunidad campesina Huayana, donde se observó un debilitamiento en su organización. Para el año 2017, se redujeron no solo para el caso de la comunidad campesina de Huayana, sino también, para las otras comunidades campesinas. Ello se demuestra en la disminución de quejas, sanciones por incumplimiento de acuerdos comunales $u$ ordenanzas municipales en aspecto a linderos, apropiaciones, restricción de ganado, cuidado y siembra de agua. Asimismo, cabe destacar que, en los territorios estudiados, no se tiene presencia o instalación de algún puesto policial. Estos resultados muestran que existe un estado fuerte, mayor integración, respeto y compromiso de ejecutar los acuerdos comunales.

Los resultados de los objetivos específicos se muestran a continuación.

Para la producción orgánica, en los ratios de beneficio monetario de la producción orgánica, la mayoría demuestran que los costos son cubiertos y existe una utilidad. Es decir, los ingresos superan los costos (sin impuestos, porque los productos agrarios, pecuarios, están exonerados en la región sierra del Perú). A pesar de la variabilidad de precios y costo de la mano de obra diversa, han descendido los ratios de beneficio monetario de la producción orgánica. Para el año 2017 cayeron respecto al año 2013, cuyo rango de variación fue 1.4 a 2.33, respectivamente. Ello se debe a la caída de precios, ceñido a la temporada de siembra y la sobreproducción (cosecha), lo que no permitió sostener precios.

Estos resultados se reflejan a partir de la decisión de mantener la producción orgánica, apoyarse a comercializar en forma directa a través de vínculos familiares y parentesco, lo cual hace posible garantizar el pacto de venta $u$ otros casos a través de instituciones que buscan empoderar la cultura andina y la organicidad de sus productos, afianzando la comercialización. Es el caso de las comunidades campesinas de Pomacocha y Huayana, donde existió la presencia de un proyecto que fortaleció la recuperación de saberes ancestrales y la comercialización de la diversidad del tubérculo papa, lo que se concretó mediante contratos pre establecidos, donde el precio no solo alcanza el valor de producción, además contiene el pago por mantener prácticas de organicidad y preservar el medio ambiente, es decir un comercio justo. Al año 2017, las comunidades de San Miguel de Chaccrampa y Santiago de Yaurecc han iniciado actividades para imitar lo sucedido en las comunidades campesinas de 
Pomacocha y Huayana en el año 2013.

Los años 2013 y 2017, las comunidades campesinas Matapuquio, Belen Anta, Santiago de Paucar, que conforman la microcuenca 3, fueron ubicas por encima del indicador $1.90 \mathrm{en}$ las variaciones con descenso; esto significa que por cada S/ 1.00 se obtiene S/ 0.90 de beneficio. Con respecto a las microcuencas 1 y 2 para los años 2013 y 2017, las variaciones con descenso las ubicaron por encima del indicador 1.4 , significa que por cada $\mathrm{S} / 1.00$ se obtiene $\mathrm{S} / 0.40$ de beneficio. Para las microcuencas 1 y 2, las diferencias de beneficio son altas con respecto a la microcuenca 3 .

Tanto para el año 2013 y 2017, el cultivo temporal frecuente en la zona alta en las tres microcuencas fue el tubérculo papa, seguido por la quinua, olluco y mashua. El cultivo temporal frecuente en la zona media de las tres microcuencas fue maíz amiláceo, seguido por haba y papa, con algunas excepciones de plantaciones forestales. El cultivo permanente frecuente en la zona baja de las tres microcuencas son las plantaciones de frutales $\mathrm{y}$, en algunos casos, forestales. Para los años 2013 y 2017, el costo de la mano de obra ha sufrido variaciones considerables, debido a la mayor presencia de la minería artesanal en alianza con los dueños de los denuncios mineros. En la mayoría de indicadores para el año 2017, el resultado alcanza un $50 \%$ superior, respecto los indicadores y resultados para el año 2013.

Con respecto al uso de los recursos naturales, la cantidad porcentual de servicios de saneamiento básico, para los años 2013 y 2017, se ha mejorado la cobertura en beneficio de la población (agua entubada y clorada, y desagüe). Los resultados para los años 2013 y 2017 arrojan que son heterogéneos, no extremos; porque encontramos sistemas paralelos en los casos de desagüe; primero por arrastre hidráulico y el segundo, por vierta de hollín. La tendencia es homogenizar los servicios de evacuación por arrastre hidráulico, mejorando las condiciones de equidad de uso y satisfacción. Se programa faenas de limpieza, uso racional y minimización de la pérdida del agua, para el caso de agua de riego. Asimismo, en ciertas microcuencas existen deficiencias en almacenar el recurso agua, existen sequías debido al bajo volumen de lluvia. En los años 2015 y 2016, se iniciaron proyectos presupuestados, para mejorar el almacenamiento y cuidado del agua, fue el caso de las comunidades campesinas de San Miguel de Chaccrampa, Huayana, Belén Anta y Matapuquio. Esta organización y gestión de proyectos permitieron minimizar el estado de alta vulnerabilidad en salud, ante la decisión de destinar una proporción de volumen de recurso agua para el arrastre hidráulico de desechos sólidos (desagüe), con esta decisión se enfrentó a la escasez del recurso hídrico.

Para los años 2013 y 2017, las buenas prácticas de cuidar, proteger áreas naturales, basadas en la reciprocidad y uso común, conocida como Ayni, se fortalecieron. Para el año 2013, la práctica del Ayni tuvo una presencia de regular a fuerte en las comunidades; siendo la comunidad campesina de Chiara, el único caso cuya presencia era débil; debido a la influencia de la formalización de la explotación minera por asociación de los mineros artesanales y el propietario de la concesión minera. Para el año 2017, las prácticas de reciprocidad cambian en las microcuencas 1 y 3 , desde un estado fuerte hacia regular; significa que se debilitó esta práctica ancestral. Por el contrario, se fortalece la libre disposición e intervención de pagar por servicio prestado.

Los resultados respecto a las prácticas de siembra y conservación del agua, para los años 2013 y 2017, fueron diferentes. Para el año 2013, en las microcuencas 2 y 3 , se constató que entre una y dos comunidades campesinas realizaban la práctica de siembra y conservación de agua. Para el año 2017, en las mismas cuencas, se observa un cambio, se extiende la práctica de siembra y conservación del agua; como ejemplo se tiene a la comunidad campesina de San Miguel de Chaccrampa en la microcuenca 2 y a las comunidades campesinas Belén Anta y Santiago de Paucar en la microcuenca 3 .

Con respecto a la cultura a partir de las prácticas de protección y conservación del suelo en las áreas naturales protegidas por la comunidad campesina; para el año 2013, se observa que son protegidas entre el $50 \%$ al $100 \%$ del 
territorio, existe un plan de conducción aprobado donde el espacio es destinado a plantas forestales por excelencia, en algunos casos separado o asociado de pastos naturales y frutales; además se apoyan en mantener la práctica de organicidad que tiene una antigüedad entre 30 a 50 años. Para el año 2017, se mantienen estos resultados en las tres microcuencas de estudio. Además, se ampliaron las indagaciones respecto a la existencia de ciertas especies, frecuentemente observadas y que interactúan con el hombre. Los resultados indican que están desapareciendo; en las microcuencas 1 y 2 existe peligro de extinción alto, por motivo de expansión de frontera agrícola y caza furtiva; que origina reducción del espacio de alimentación y reproducción de las especies como el zorro, puma y venado. Para la microcuenca 3 existe peligro de extinción medio. Por otro lado, se puede determinar que existe un nivel de bienestar fuerte en el uso adecuado de los recursos naturales, para el caso agua, tierra y forestal.

Referente a la gobernanza comunal, para los años 2013 y 2017, se observa que no existen cambios o modificaciones sustanciales en la Ley 24656 y Ley 24657, además en la Constitución Política del Perú, está tácito y definido el proceso de reversión de los territorios comunales cuando pierden o desaparecen las condiciones por las que se conforma una comunidad campesina, y donde los territorios comunales campesinos serían revertidos al Estado Peruano. La organización de las comunidades campesinas se ampara en las normas expuestas y la existencia de estatutos y reglamentos; los cuales norman el quehacer comunal, prueba de ello es el D.S. 091-TR. Los acuerdos comunales son propuestos por la directiva comunal, estos pasan por un proceso de análisis, para posteriormente ser aprobados o rechazados por la asamblea comunal. Si bien los resultados arrojan heterogeneidad, solo en la comunidad campesina Huayana se evidencia un debilitamiento en su organización para el año 2013; por las quejas, sanciones por incumplimiento de acuerdos comunales en aspecto de linderos, apropiaciones, restricción de ganado, cuidado y siembra de agua. Para el año 2017, la situación cambia, para el caso de las comunidades campesinas de Huayana y Santiago de Yaurecc, con tendencia en las tres microcuencas de estudio, existe una mayor integración, respeto, compromiso y legitimar las disposiciones aprobadas por la asamblea general comunal en beneficio de todos. Asimismo, cabe destacar que en los territorios estudiados no se tiene presencia o instalación de algún puesto policial, a pesar que años atrás (década 90 del siglo XX), en varios distritos que contienen a las comunidades campesinas, estuvieron alguna vez instalados puestos policiales. La respuesta recibida es que el accionar del policía interfería o distorsionaba la función de las autoridades comunales; no fortalecía ni legitimaba la función del directivo comunal. Por ello, fue conveniente la decisión en consenso que se retirara, esto ocurrió en la primera década del siglo XXI. El nivel de gobernanza alcanzado es fuerte debido a las pocas o mínimas quejas, sanciones por incumplimiento de acuerdos $\mathrm{u}$ ordenanzas municipales en aspecto ambiental, cuidado y siembra de agua, que se comprueba desde el año 2013 al año 2017. Existe explotación de recursos minerales a nivel informal - artesanal desde el año 2012 en las comunidades campesinas de Tumayhuaraca y Chiara. A partir del año 2015, aparece explotación de recursos mineros en las comunidades campesinas de Huayana y San Miguel de Chaccrampa. La forma de aprovechamiento de los recursos naturales minerales es el convenio y contrato de explotación entre una asociación de mineros artesanales que representa a la comunidad campesina y el propietario del denuncio minero. La forma de aprovechamiento solo cumple con los requisitos mínimos necesarios, porque existe evidencia de problemas en la explotación del recurso. Podemos concluir que se administra en forma coordinada entre los pobladores, empresa minera y autoridades comunales; donde la licencia social, servidumbre y oferta laboral son consensuadas en asamblea comunal, aprobado-cumplido por la empresa minera. Por ello, desde el año 2013 al 2017, podemos determinar que existe un fuerte estado de gobernanza comunal. 


\section{CONCLUSIONES}

Existe un estado de bienestar colectivo comunal fuerte los años 2013 y el 2017, que se sostiene en la cultura de uso adecuado de los recursos naturales: el caso saneamiento, tierra y áreas naturales protegidas, se concluye que se protege y conserva de forma eficiente, cuyo nivel fue de aceptable a óptimo (por encima del $66 \%$ hasta alcanzar el $90 \%$ ), las cifras nos demuestran incrementos entre los años comparados. Las áreas naturales protegidas se conservan, protegen por acciones de la propia comunidad; además, existen planes de manejo monitoreados, para ambos años de estudio se mantiene las actividades constantes. Por otro lado, el ratio de beneficio de producción orgánica para los dos años es superior a 1.4, pero sufre descenso para el año 2017. La gobernanza comunal es aceptable en el año 2013, y el año 2017 es considerada óptima; esto debido a la reducción de quejas, denuncias, conflictos, acuerdos y consenso de explotación minera, entre otros. Además, esta gobernanza comunal es suficiente y no se necesita ningún respaldo policial.

\section{REFERENCIAS BIBLIOGRÁFICAS}

Banco Interamericano de Desarrollo (2009), Manual de Buenas Prácticas de Agencias de Desarrollo Regional en América Latina y Europa. Borrador de Informe Final. Diciembre.

Constitución Política del Perú (1993). Diario Oficial El Peruano.

Decreto Supremo No 091-1991-TR. (1991). Reglamento General de Comunidades C a m pesin as ( publi ca do 1991/febrero/15). Diario Oficial El Peruano.

FAO (2001). Pesticidas, residuos en los alimentos. Roma 2002.
Figueroa, C. (2006). Evaluación agroecológica de las experiencias de los agricultores de manzana con predios ecológicos o integrados, Valle de Mala-Perú. (tesis de maestría). Universidad de Andalucía, España.

Garza, M. (2004). Percepeción del paisaje matorral xerófilo en relación a las actitudes hacia el mismo, por alumnos del sexto grado de primaria, Municipio Baja California. Monterrey. Universidad Autónoma de Monterrey.

Instituto Nacional de Estadística e Informática Perú (2018).

Ley $\mathrm{N}^{\circ}$ 24656. Ley General de Comunidades Campesinas. (publicada el 1987/marzo/30). Diario Oficial El Peruano.

Ley $\mathrm{N}^{\circ}$ 26821. Ley Orgánica para el aprovechamiento sostenible de los recursos naturales. (publicada el 1997/junio/25). Diario Oficial El Peruano.

Ley $N^{\circ} 24657$. Ley de Deslinde y Titulación de Territorios Comunales. (publicada el 1987/abril/14). Diario Oficial El Peruano.

MINISTERIO DEL AMBIENTE. (2011). Plan Nacional de Acción Ambiental. Planaa Perú 2011-2021. Lima: Minam.

Organización de las Naciones Unidas (2016). Gobernanza. Recuperado de http:///www.org/es/globalissues/g overnance/.

Ostrom, E. (2009). A General Framework for Analyzing Sustainability of SocialEcological Systems. Science, 325(24) 419-422. Recuperado de https://ils.indiana.edu/

Palomino, G. (2011). Investigación cualitativa y cuantitativa en Educación. PunoPerú. 